ISSN-i 1390-7042 | IssN-e 2602-8484

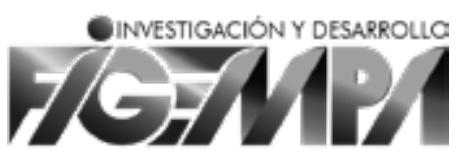

\title{
MODELOS DE DEPÓSITOS TIPO VHMS PARA LAS DIVISIONES DE LA CORDILLERA REAL: ALAO Y SALADO
}

\begin{tabular}{ll}
\hline \multicolumn{2}{c}{ VHMS deposit models for the cordillera Real divisions: Alao and Salado } \\
\hline Kevin D. Calero & Laura J. Albacura \\
kdcalero@uce.edu.ec & ljalbacura@uce.edu.ec \\
Universidad Central del Ecuador & Universidad Central del Ecuador \\
ORciD: https://orcid.org/0000-0003-4888-8056 & \\
Stalin Solano & Francisco Viteri \\
spsolano@uce.edu.ec & frviteri@uce.edu.ec \\
Universidad Central del Ecuador & Universidad Central del Ecuador \\
ORcID: https://orcid.org/0000-0002-1187-5780 & \\
\hline
\end{tabular}

Recibido: octubre de 2020

Aprobado: mayo de 2021

ARTÍCULO ORIGINAL

DOI: $10.29166 /$ revfig.vli2.2664 palabras Clave Cordillera Real, vhms, evolución, tipo Beshi, división Salado.

KEYWORDs Cordillera Real, vHMs, Evolution, Beshi Type, Salado.

\section{RESUMEN}

La cordillera Real comprende terrenos alóctonos y autóctonos acrecionados al continente, representados de oeste a este por las divisiones litotectónicas Guamote, Alao, Loja, Salado y Zamora. En este estudio se realiza la identificación de los posibles depósitos tipo vHMs (sulfuros masivos volcanogénicos) relacionados a las divisiones Alao y Salado, basado en estudios previos de petrología, petrografía y evolución de estas divisiones. Los parámetros que se utilizaron para comparar los tipos de modelos vHMs con los posibles depósitos de las divisiones mencionadas son: entorno geológico y tectónico, rocas encajantes, características texturales y mineralógicas de los cuerpos masivos, alteración y características geoquímicas. De esta manera se puede establecer que los depósitos de la unidad Peltetec no se asocian a modelo alguno de depósitos vHMs, al existir diferencias con respecto a la litología y geoquímica. Por el contrario, el modelo asociado a un depósito vHMs tipo Kuroko coincide con las características de un depósito vHMs en la unidad Alao-Paute. A su vez se asoció depósitos de la división Salado a un depósito de vHms tipo besshi, al encontrar similitudes en cuanto al entorno geológico-tectónico, mineralogía y semejanza de las huellas geoquímicas, sin embargo, las características de formación y evolución de la división Salado ha dificultado el desarrollo completo de estos vHMs tipo Besshi, pero no se descarta la existencia de los mismos.

\section{ABSTRACT}

The Cordillera Real comprises autochthonous and allochthones land accreted to the continent, represented from west to east by thelithothectonic divisions Guamote, Alao, Loja, Salado and Zamora Divisions. (Litherland, et al. 1994). This work describes the possible vHMs (Volcanogenic Massive Sulphide) deposits present in the Alao and Salado divisions, based on previous studies of petrology, petrography and evolution of these divisions. The parameters used to compare vHMs model types with possible deposits of the divisions are: geological environment, tectonic framework, nesting rocks, associated rocks, textural and mineralogical characteristics of massive bodies, alteration and geochemical characteristics. In this way, it can be established that the deposits of the Peltetec Unit are not associated with a vHMs deposit as there are differences with respect to lithology and geochemistry. In contrast, the model associated with a Kuroku-type vHMs deposit coincides with the characteristics of a vHMS 
deposit in the Alao-Paute Unit. In turn, deposits from the Salado Division were associated with a Besshi-type vHMs deposit, finding similarities in terms of the geological-tectonic environment, mineralogy and similarity of the geochemical traces, however, the formation and evolution characteristics of the Salado Division has hindered the full development of these Besshi-type vHMs, but their existence is not ruled out.

\section{INTRODUCCIÓN}

En la cordillera Real del Ecuador (ver Figura 1), se han identificado divisiones litotectónicas definidas de oeste a este como las divisiones Guamote, Alao, Loja, Salado y Zamora (Litherland et al., 1994), sus edades varían desde el Paleozoico hasta el Cretácico y su morfología es el resultado de la tectónica compresiva de una alternancia de terrenos alóctonos y autóctonos. Dentro de estas divisiones acrecionadas se han realizado varios estudios con respecto a evidenciar el potencial geológico-minero y probable desarrollo económico de la región, tomando en cuenta los rasgos morfoestructurales y los principales depósitos emplazados identificados en la cordillera, como los pórfidos de $\mathrm{Cu}$-Au y epi-mesotermales relacionados con el emplazamiento plutónico y los depósitos de sulfuros masivos volcanogénicos (vhms por sus siglas en inglés).

Los depósitos de sulfuros masivos volcanogénicos han sido poco explorados y/o desarrollados y considerados menos rentables por su tamaño, razón por la que no se han realizados programas de investigación a detalle en la cordillera Real.

\section{CARACTERÍSTICAS GENERALES DE LOS DEPÓSI- TOS VHMS}

La concentración de los VHMS se centra en plataforma oceánica, su particularidad es la evidencia de fumarolas/chimeneas submarinas ricas en sulfuros, en ocasiones se han formado por emplazamiento en los sedimentos cercanos a la superficie. Estos depósitos están constituidos por masas de varios tamaños de sulfuros masivos, entendiendo por masivos que contengan más del 60\% de sulfuros en el total de la masa mineral (PRODEMINCA, 2000).

Su importancia en la minería es grande y destacan algunos aspectos que realzan su valor económico. Entre ellos están:

1. La diversidad de metales ( $\mathrm{Cu}, \mathrm{Pb}, \mathrm{Zn}, \mathrm{Ag}, \mathrm{Au}, \mathrm{Fe}$, $\mathrm{Cd}, \mathrm{Sb}, \mathrm{Se}, \mathrm{Co}, \mathrm{Bi}, \mathrm{Sn}, \mathrm{Hg}$, entre otros).

2. No son complejos para el proceso de extracción minera, al tratarse de masas de sulfuros con poca ganga facilita su explotación a cielo abierto siempre y cuando no tengan una sobrecarga potente de secuencias volcánicas cuaternarias o de otro tipo litológico.

3. Recuperación de varios metales al existir enriquecimientos supergénicos de gran valor en oro.

4. La existencia de grupos de varias masas situadas en distritos restringidos, lo que facilita su exploración y reduce los gastos de explotación (PRODEMINCA, 2000).

Con esta referencia bibliográfica se podrá generar planes de exploración para determinar la posible existencia de depósitos relacionados a las divisiones Alao y Salado, haciendo alusión a su litología y a la favorable génesis de acuerdo con su evolución cuyos límites orientales marcan paleo-suturas de subducción jurásico-cretácicas.

\section{METODOLOGÍA}

El presente estudio se refiere a un análisis e investigación bibliográfica, por lo cual se recopiló información que nos permita entender la evolución, el entorno litológico y la tectónica de cada división; además de las características generales de los modelos vHMs.

El análisis de la información se realizó de forma comparativa entre depósitos tipo, ambientes marinos similares y la lito-estratigrafía de cada uno. La información recopilada se utilizó para interpretar y comparar las características generales de los principales modelos de vHMs con las divisiones más propicias para presentar estos depósitos.

\section{CONTEXTO GEOLÓGICO GEOLOGÍA REGIONAL}

\section{División Alao (un arco de islas oceánico del Jurá- sico medio)}

La división Alao comprende una secuencia de rocas metavolcánicas y metasedimentarias de edad jurásica diferenciadas por las unidades metamórficas Peltetec, Maguazo, Alao-Paute, y El Pan, aflorantes en las estribaciones occidentales de la cordillera y en ventanas dentro del valle Interandino (Litherland, et al., 1994) (ver Figura 2).

La unidad Peltetec es un complejo ofiolítico desmembrado, conformado por gabros, serpentinitas, basaltos y se ha interpretado como un melange tectónico aflorante a lo largo de la vía Licto-Alao, sector 
en el que se ha descrito metagabros y metabasaltos. $\mathrm{Al}$ este está limitada por la unidad Maguazo y al oeste por pizarras y filitas de la unidad Punín y Cebadas de la división Guamote. Hacia el norte del poblado de Peltetec, la secuencia ofiolítica está expuesta a lo largo del río Quishpe y alrededor de Penipe. A lo largo del río Blanco se ha identificado metabasaltos, serpentinitas, piroxenitas, hornblenditas, filitas negras y rocas volcanoclásticas (Litherland et al., 1994).

Spikings et al. (2015), mediante dataciones en metabasaltos y gabros por el método ${ }^{40} \mathrm{Ar} /{ }^{39} \mathrm{Ar}$, establecieron la edad de esta unidad como Cretácico temprano $(134,3 \pm 12,8$ Ma y $134,7 \pm 0,9 \mathrm{Ma})$.

Rocas de la unidad Maguazo están representadas por turbidíticas y secuencias volcánicas (andesita-basalto) ligeramente metamorfoseadas, pizarras carbonáceas, ortocuarcitas y cherts (Aspden y Litherland, 1992). La presencia de fósiles en esta unidad señala una edad jurásica, interpretada como una secuencia marina (Litherland et al., 1994).

En la unidad Alao-Paute, conformada por esquistos cloríticos y rocas verdes de composición andesítica-basáltica, ubicada al noreste de Cuenca, afloran metasedimentarias, filitas grafíticas-cuarcíferas y rocas con tremolita-clinozoisita (Aspden et al., 1992). Dataciones de ${ }^{206} \mathrm{~Pb} /{ }^{238} \mathrm{U}$ en circones presentan una edad de 163,7 \pm 1,6 Ma (Spikings et al., 2015).

La unidad El Pan se presenta como un cinturón elongado de $70 \mathrm{~km}$ de largo y hasta $7 \mathrm{~km}$ de ancho, con rumbo andino (NE-SO), litológicamente representada por esquistos cloríticos, grafitosos, filitas y rocas de protolito ígneo, verdes más masivos con actinolita \pm clinozoisita \pm epidota. Es considerada como una secuencia de tras-arco, del arco de islas Alao-Paute y de edad jurásica (Litherland et al., 1994).

\section{MODELO EVOLUTIVO DE LA DIVISIÓN ALAO}

Se ha interpretado un evento tectónico entre 170-180 $\mathrm{Ma}$, probablemente relacionado con la formación de un arco volcánico in situ con polaridad al oeste, formado al borde noroccidental de la placa continental sudamericana como lo manifiestan Cochrane (2013) y Spikings et al. (2015). En dicho arco se desarrollaron varios episodios volcánicos que dieron origen a una sucesión de rocas esencialmente basálticas y volcanosedimentarias de componentes andesíticos.

Litherland et al. (1994), describe que las rocas de la división Alao se formaron por un arco de islas oceánico durante el Jurásico medio y de origen alóctono, que posteriormente fue acrecionado al continente. Cochrane (2013) y Spikings et al. (2015), interpretan a esta unidad como parte de un arco formado sobre una zona de subducción buzando al este, a lo largo de una franja adelgazada de un margen continental, dando lugar a rocas volcánicas máficas isotópicamente jóvenes y en cuyo arco alberga grandes volúmenes de areniscas ricas en cuarzo-circón-turmalina, además de edades $\mathrm{U} / \mathrm{Pb}$ de los circones detríticos que revelan una derivación del cratón sudamericano.

\section{División Salado (litologías de arco de islas y plu- tónicas)}

La división Salado comprende rocas volcánicas basálticas, metamorfoseadas y metasedimentarias, se encuentra limitada al este por la falla Cosanga-Méndez y al oeste por la falla Llanganates, la cual la separa de la división Loja (ver Figura 3) (Litherland et al., 1994). Se ha diferenciado las unidades Upano, Cuyuja y Cerro Hermoso y, además, se incluyen rocas plutónicas metamorfoseadas de los granitoides de azafrán.

La unidad Upano comprende rocas verdes andesíticas, esquistos cloríticos y metagrauwacas intercalados con esquistos pelíticos y grafitosos, las cuales forman un cinturón casi continuo de hasta $15 \mathrm{~km}$ a lo largo del borde oriental de la cordillera Real. El grado metamórfico varía de medio a bajo. La sección tipo está ubicada en la carretera Guamote-Macas en la margen derecha del río Upano. Existen afloramientos a lo largo de la carretera Papallacta-Baeza y en las secciones de los ríos Upano, Cosanga y Oyacachi. Los datos geocronológicos y paleontológicos le asignan una edad Mesozoica (Litherland et al., 1994). La edad promedio de palinoflora (perinopollenites elatoides) en filitas indican una edad Jurásico inferior-Cretácico, mientras que una datación K/Ar tiene como resultado una edad de $54 \pm 2$ Ma considerada como una edad de reajuste (Litherland et al., 1994). Dataciones realizadas por Cochrane (2013), indican una edad $206 \mathrm{~Pb} / 238 \mathrm{U}$ de $121,0 \pm 0,8 \mathrm{Ma}$.

La unidad Cuyuja ocurre al norte de la cordillera Real, está expuesta en el sector de Cuyuja (sobre la carretera Papallacta-Baeza) y forma un cinturón de hasta $10 \mathrm{~km}$ de ancho dentro del denominado complejo de cabalgamientos (nappes) Cuyuja. Su litología comprende esquistos con grafito y moscovita, esquistos pelíticos, e intercalaciones de esquisto verde. Las edades obtenidas mediante el método de K/Ar en moscovitas y biotitas son $82 \pm 3 \mathrm{Ma}$ (Kennerley, 1980 en Litherland et al., 1994) y $59 \pm 2 \mathrm{Ma}$ (Herbert \& Pichler, 1983 en Litherland et al., 1994), pero en la unidad Upano son consideradas como edades de reajuste. Sin embargo, su relación genética con el adyacente plutón Azafrán expondría para esta unidad una edad jurásica (Litherland et al., 1994).

La unidad Cerro Hermoso es una secuencia carbonatada que aflora en un cinturón estrecho en Cerro 
Figura 1. Mapa de ubicación de la cordillera Real. Los terrenos de la cordillera Real están comprendidos de grupos litológicos con edades que varían desde el Paleozoico hasta el Cretácico, representados de oeste a este por las divisiones Guamote, Alao, Loja, Salado y Zamora.

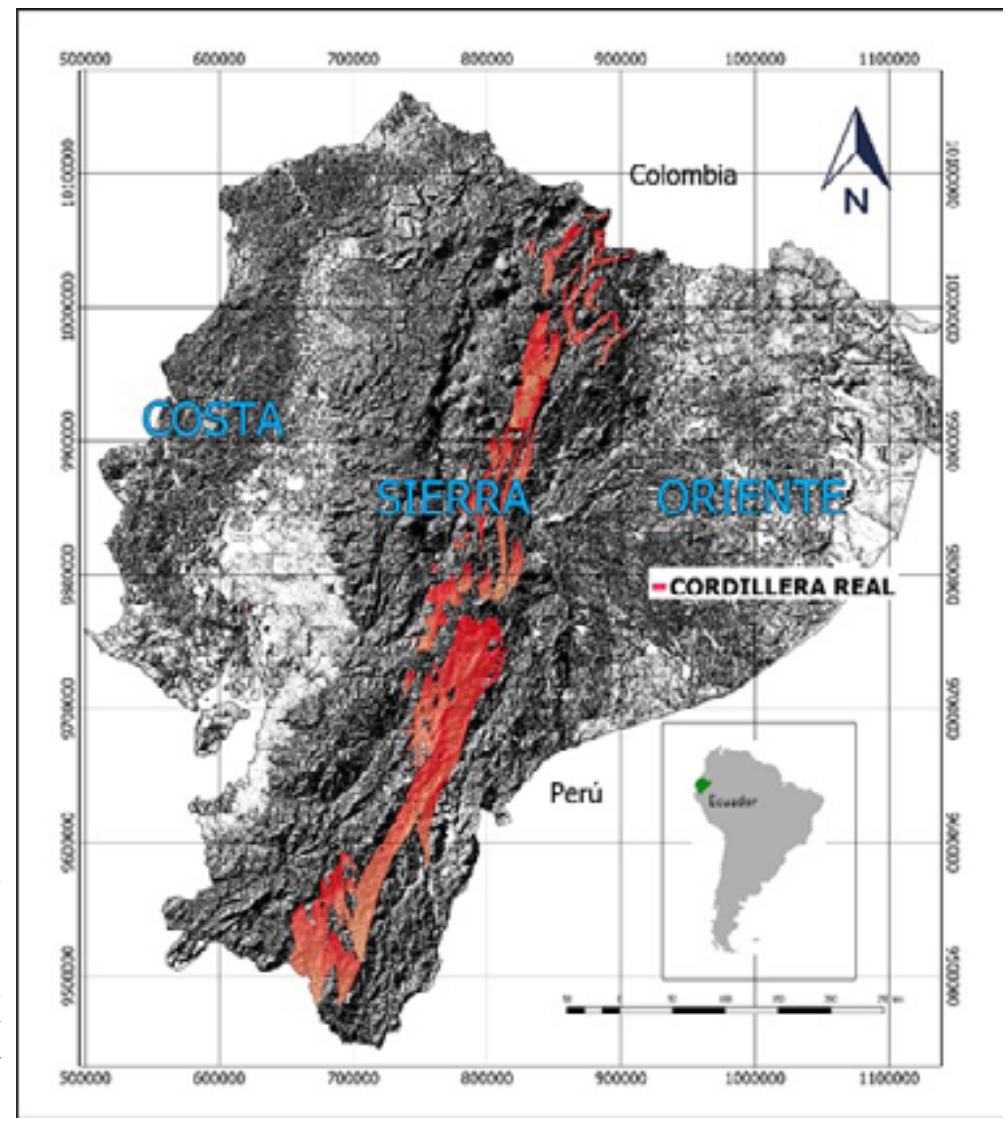

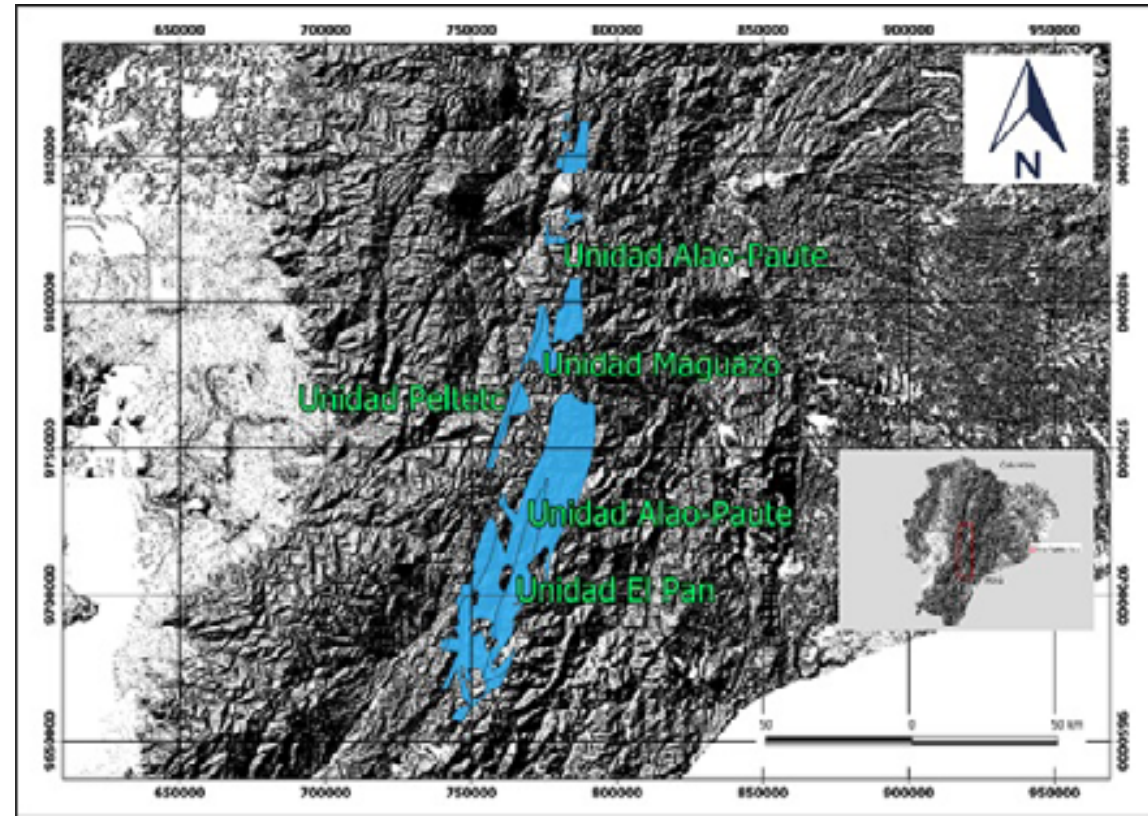

Figura 2. Mapa de ubicación y distribución de la unidad Alao en la cordillera Real. Este terreno comprende una secuencia de rocas metavolcánicas y metasedimentarias, pertenecientes a las subdivisiones metamórficas Peltetec, Alao-Paute, El Pan y Maguazo de edad jurásica.
Hermoso. Comprende un espesor de unos $450 \mathrm{~m}$ de calizas negras metamorfizadas, filitas calcáreas negras y calco-arenitas, tiene un fuerte clivaje y microscópicamente presenta evidencias de fragmentos de concha y biotita recristalizados. Está intruida por el plutón de Azafrán y la edad que se le asigna es Jurásico tempra- no a medio (Litherland et al., 1994).

La unidad Azafrán constituye un cinturón de granitoides deformados, representados en el norte por los plutones Chingual y Sacha, en el sur por Azafrán. Su litología consiste en granodioritas y tonalitas hornbléndicas y biotíticas, de grano medio a grueso, 
variablemente deformadas y gnéisicas, además de estar presentes dioritas, hornblenditas y gabros. Varias dataciones por diversos métodos dan edades dependiendo de la deformación de las rocas como $50 \mathrm{Ma}$ por K/Ar para una metadiorita, $120 \pm 5 \mathrm{Ma}$ por $\mathrm{Rb} /$ Sr para un metagranito y $142,7 \pm 2,9 \mathrm{Ma}$ por $\mathrm{U} / \mathrm{Pb}$ en zircones de granitos (Litherland et al., 1994). Análisis recientes muestran edades $\mathrm{U} / \mathrm{Pb}$ de $140,7 \pm 0,7 \mathrm{Ma}$ y $143,5 \pm 1,3 \mathrm{Ma}$, probablemente corresponde a la edad de la intrusión, en tanto las restantes se refieren posiblemente a eventos metamórficos tanto regionales como de contacto (Cochrane, 2013).

\section{MOdelo eVolutivo de LA división SAlado}

Tras la generación de granitos anatécticos tipo $\mathrm{S} y$ la apertura del rift triásico, relacionado a una fase extensiva donde se evidencia discordancias entre las capas rojas (reds beds) de la formación Sacha y los sedimentos marinos de la formación Santiago en la cuenca oriente, se inicia una etapa de volcanismo intraformacional durante gran parte del Jurásico con la generación del arco volcánico Misahuallí, asociada con actividad plutónica desarrollando cuerpos magmáticos tipo I (Aspden \& Litherland, 1992). Este fenómeno está relacionado con un cambio en el marco geodinámico, el cual corresponde al arranque de la subducción andina evidenciada por el volcanismo calco-alcalino Misahuallí (Baby et al. 2004).

La división Salado interpretada por Litherland et al., (1994) como un arco volcánico-plutónico situado junto al margen continental, probablemente fue contemporáneo con el arco continental de Misahuallí al este. La actividad volcánica generó tobas y flujos andesíticos en un ambiente marino inestable con subsistencia turbidítica relacionada y con periodos más estables de formación de carbonato marino. Los eventos ígneos y sedimentarios de la división Salado parecen relacionarse al Jurásico medio/superior.

\section{DISCUSIón}

\section{DIVISIÓN ALAO-UNIDAD PELTETEC (DEPóSito DE SULFUROS MASIVOS TIPO CHIPRE)}

Litherland et al. (1994), definen el entorno geológico de la unidad Peltetec como una secuencia ofiolítica, con metabasaltos, serpentinitas, piroxenitas, hornblenditas, filitas negras y volcanoclásticas, este entorno geológico coincide con el entorno geológico de los depósitos de sulfuros masivos tipo Chipre, definidos por Singer \& Mosier (1986), que corresponden a una asociación ofiolítica (basaltos almohadillados, diques de diabasas, dunitas y harzburgitas tectonizadas y rocas metasedimentarias ) (tabla 1).
Singer y Mosier (1986), definen que el marco tectónico de un depósito de sulfuros masivos volcanogénicos tipo Chipre corresponde a estructuras de graben centro-oceánicos o extensionales tras-arco, esto se diferencia del marco tectónico de la unidad Peltetec, al ser interpretado por Litherland et al. (1994), como una sutura de acreción del terreno Alao en el evento Peltetec-Palenque, además se observan esfuerzos de transpresión y cabalgamientos.

Singer y Mosier (1986), establecen que las rocas encajantes en los depósitos tipo Chipre son basaltos almohadillados, brechas volcánicas básicas y sedimentos de grano fino, mientras que las rocas encajantes de la unidad Peltetec caracterizadas por Litherland et al. (1988), son areniscas verdes, pizarras negras y turbiditas, esto es un contraste para el modelo propuesto para esta unidad, ya que la litología es una secuencia sedimentaria de erosión de la proto-cordillera, para que este modelo se cumpla es necesario una roca encajante básica, asociado a piso oceánico con Cherts ricos en Fe y Mn.

Otros puntos que desfavorecen el modelo propuesto para la unidad Peltetec son las características texturales-mineralógicas, porque en la unidad Peltetec definida por Litherland et al., (1988) no se encuentran sulfuros masivos o diseminados; y la alteración presente es una serpentinización, mientras que en los depósitos de sulfuros masivos volcanogénicos tipo Chipre, definido por Singer y Mosier (1986), son abundantes cuarzo, calcedonia y clorita, junto con algo de illita talco y calcita.

Singer y Mosier, (1986) establecen que las características geoquímicas de un depósito de sulfuros masivos tipo Chipre, corresponden a la pérdida de Ca y $\mathrm{Na}$, introducción y redistribución del Mn y Fe en la zona de vetillas mineralizadas (stockwork), mientras que en la subdivisión Peltetec presenta $\mathrm{Cu}-\mathrm{Ni} \pm \mathrm{Co}$, $\mathrm{Cr}$ (ofiolita), Au (zona cizallada con vetas de cuarzo mesotermales) y polimetálicos (klippes de skarn) (PRODEMINCA, 2000) (ver Tabla 1).

$\mathrm{Al}$ comparar las características de la unidad con el modelo de depósito de VHMs, éstas son coincidentes en el contexto geológico, pero difieren con respecto a las rocas de caja, características texturales y mineralógicas de los cuerpos masivos de sulfuros, la alteración y características geoquímicas.

\section{DiVisión ALAO, UNIDAd ALAO-PAUTE (OCU- RRENCIA DEPÓSITO DE SULFUROS MASIVOS TIPO KUROKO)}

Singer (1986); Singer y Mosier, (1986), caracterizan el entorno geológico de un depósito de sulfuros masivos tipo Kuroko, el cual corresponde a una secuencia vol- 
Tabla 1. Comparación de características geológicas

\begin{tabular}{|c|c|c|}
\hline Características & vнмs tipo Chipre & Unidad Peltetec \\
\hline Entorno geológico & Asociación ofiolítica & Secuencia ofiolítica \\
\hline Marco tectónico & $\begin{array}{l}\text { Grabens centro-oceánicos o extensio- } \\
\text { nales tras-arco }\end{array}$ & $\begin{array}{l}\text { Acreción del terreno Alao en el even- } \\
\text { to Peltetec-Palenque con corrimien- } \\
\text { tos/ transpresión }\end{array}$ \\
\hline Rocas encajantes & $\begin{array}{l}\text { Basaltos almohadillados, brechas vol- } \\
\text { cánicas básicas y sedimentos de grano } \\
\text { fino }\end{array}$ & $\begin{array}{l}\text { Areniscas verdes, pizarras negras y } \\
\text { turbiditas }\end{array}$ \\
\hline $\begin{array}{l}\text { Características texturales y minera- } \\
\text { lógicas de los cuerpos masivas }\end{array}$ & $\begin{array}{l}\text { Sulfuros dominantes: pirita } \pm \text { calcopi- } \\
\text { rita + esfalerita + magnetita Brechifica- } \\
\text { dos y recementados }\end{array}$ & $\begin{array}{l}\text { No presenta cuerpos masivos de } \\
\text { sulfuros }\end{array}$ \\
\hline Características geoquímicas & $\begin{array}{l}\text { Perdida de Ca y Na. Introducción y } \\
\text { redistribución del Mn y Fe en la zona } \\
\text { de «stockwork» }\end{array}$ & $\begin{array}{l}\mathrm{Cu}-\mathrm{Ni} \pm \mathrm{Co}, \mathrm{Cr} \text { (ofiolita), Au (zona } \\
\text { cizallada con vetas de cuarzo meso- } \\
\text { termales), Polimetálicos (klippes de } \\
\text { skarn) }\end{array}$ \\
\hline
\end{tabular}

Basado en Singer (1986a); Singer y Mosier (1986); Hoy (1995b), Litherland et al. (1994).

cano-sedimentaria con características de arco de islas lo cual corresponde ampliamente al entorno evolutivo del arco de islas Alao, definido por Litherland et al. (1988).

El marco tectónico de un depósito de sulfuros masivos tipo Kuroko, definido por Singer, 1986c; Singer y Mosier (1986b), corresponde a un arco de islas y, dentro de ellos, sectores con actividad tectónica extensional, mientras que la división Alao, el marco tectónico definido por Litherland et al. (1988), corresponde a un arco de islas acrecionado al continente con una tectónica transpresional, el cual ha sufrido una intensa deformación debido a la falla frente Baños y una tectónica regional de fallas posteriores de rumbo E-NE (tabla 2).

Las rocas encajantes de un depósito de sulfuros masivos tipo Kuroko son volcánicas submarinas de composición félsica a intermedia (Singer, 1986c; Singer y Mosier, 1986b), mientras que las rocas encajantes de la unidad Alao-Paute, son esquistos verdes y metavolcanitas con albita+epidota+clorita \pm cuarzo (Litherland et al., 1994). Las rocas asociadas de estos depósitos coinciden en cuanto a secuencias básicas, andesíticas y sedimentarias.

Singer (1986); Singer y Mosier (1986), establecen las características texturales y mineralógicas de los cuerpos masivos de un depósito de sulfuros masivos tipo Kuroko. En la zona superior encontramos: pirita
+ esfalerita + calcopirita \pm pirrotina \pm galena \pm barita \pm tetraedrita + bornita, en las zonas marginales se ha identificado yeso/anhidrita, y en la zona inferior pirita + calcopirita \pm esfalerita \pm pirrotina \pm magnetita. La textura es bandeada con pliegues de «slumping».

Las características texturales y mineralógicas de los cuerpos masivos en los depósitos de la Unidad Alao-Paute, caracterizados por Litherland et al. (1994), son cuerpos con una recristalización intensa, transformando a la pirita en cristales idiomorfos y subidiomorfos, sin embargo, se puede distinguir un bandeamiento paralelo. También se encuentra pirita masiva con una foliación $\mathrm{N}$-s. Los cuerpos presentan del 60 al $95 \%$ de sulfuros (pirita \pm calcopirita \pm enstantita \pm esfalerita \pm bornita), bajos niveles de magnetita y los minerales que constituyen su matriz son cuarzo, sericita, moscovita y barita.

Singer (1986); Singer y Mosier (1986), establecen que las características geoquímicas de un depósito de sulfuros masivos tipo Kuroko son $\mathrm{Au}$ y $\mathrm{Pb}$ altos en el gossan; enriquecidos en $\mathrm{Mg}$ y $\mathrm{Zn}$ y diminución del $\mathrm{Na}$. En los depósitos se presentan $\mathrm{Cu}, \mathrm{Zn}, \mathrm{Pb}, \mathrm{Ba}, \mathrm{As}$, $\mathrm{Ag}, \mathrm{Au}, \mathrm{Se}, \mathrm{Sn}, \mathrm{Bi}$ y Fe. En los depósitos de sulfuros masivos de la unidad Alao-Paute encontramos $\mathrm{Fe}, \mathrm{Cu}$, $\mathrm{Pb}, \mathrm{Zn} \pm \mathrm{Ag} \pm \mathrm{Au}$; en cuanto a la alteración en estos depósitos coinciden en presentar una mineralización stockwork y alteración sericítica. (PRODEMINCA, 2000) (ver Tabla 2). 
Las características de la unidad Alao-Paute, coinciden con las características de un depósito de sulfuros masivos tipo Kuroko, esto está ampliamente descrito y corroborado por trabajos previos.

En la unidad Alao de la división Alao-Paute encontramos como ejemplo claro de depósito vHMs a Mina Pilas. Mina Pilas se encuentra ubicada a unos $15 \mathrm{~km}$ al NNe de Alao y comprende un lecho de pirita masiva intercalado entre esquisto sericítico piritizado y esquisto de clorita piritizado. La pirita es aurífera $y$, aunque no se registran valores de metales base en el ensayo, la mineralización se considera un sulfuro masivo volcanogénico, por lo tanto, la mineralización de mina Pilas podría indicar un potencial para la mineralización de vHMs de metales base en otras partes de Alao Paute (Jamielita y Bolaños, 1993).

\section{DIVISIÓN SALADO (DEPóSITO DE SULFUROS MA- SIVOS TIPO BESSHI)}

Litherland et al. (1988), definen que la división Salado es una secuencia de cuenca marginal, prisma de acreción y plataforma submarina marginal, por lo que se propone una asociación a un depósito de sulfuros masivos tipo Besshi por su afinidad. El entorno geológico de los depósitos de sulfuros masivos tipo Besshi corresponde a una secuencia sedimentaria clástica terrígena asociado con vulcanitas marinas y localmente rocas calcáreas que estarían relacionadas a las unidades Upano y Cerro hermoso.

El marco tectónico de un depósito de sulfuros masivos tipo Besshi caracterizado por Cox (1986) y Singer (1986) comprende terrenos metamórficos intensamente deformados, formación en cuencas de rift y arcos de islas o zonas traseras de arco. Al analizar la evolución de la división Salado propuesta por varios autores, se indica que corresponde a una cuenca marginal sobre un basamento continental con granitoides, que se formó durante la extensión de un rift semi desarrollado en el Triásico-Jurásico.

Cox (1986) y Singer (1986), caracterizan a las rocas encajantes de un depósito de sulfuros masivos tipo Besshi con sedimentos clásticos finamente laminados, tobas, brechas máficas tholeíticas y andesíticas con una asociación de pizarras negras, formaciones ferríticas (oxidadas) y cherts rojo, mientras que Litherland et al. (1994), definen que las rocas de la división Salado son esquistos pelíticos, metagrauvacas con asociación de rocas verdes andesíticas, esquistos verdes y metagrauvacas intercaladas con esquistos pelíticos y grafitosos (tabla 3 ).

Cox (1986) y Singer (1986), definen las características texturales y mineralógicas de los cuerpos masivos de un depósito de sulfuros masivos tipo Besshi. Los sulfuros presentes son pirita + pirrotina + calcopirita + esfalerita \pm magnetita \pm galena \pm bornita \pm tetraedrita \pm cobaltita \pm cubanita \pm estannita \pm molibdenita. En la ganga se presenta cuarzo, carbonato, albita, mica, clorita, anfibol y turmalina, finalmente la laminación es muy fina y se presenta pirita coloforme y framboidal.

En la división Salado se encuentra sulfuros diseminados predominantemente pirita, esta presenta dirección paralela a la esquistosidad (Litherland $e t$ al., 1994). La alteración en los depósitos es difícil de reconocer a causa del metamorfismo, sin embargo, se presentan zonas de cloritización (Cox, 1986; Singer, 1986).

Las características geoquímicas establecidas para un depósito de sulfuros masivos tipo Besshi (Cox, 1986a; Singer, 1986b), corresponden a una huella geoquímica de $\mathrm{Cu}, \mathrm{Zn}, \mathrm{Ag}$, Ni, Cr, Co y $\mathrm{Co} / \mathrm{Ni}>10$ halos de $\mathrm{Mn}$, mientras que en la división Salado encontramos $\mathrm{Fe}, \mathrm{Cu}, \mathrm{Pb}, \mathrm{Zn} \pm \mathrm{Ag} \pm \mathrm{Au}$ (Prodeminca, 2000) (ver Tabla 3).

De acuerdo a las características mencionadas existe concordancia en cuanto al entorno geológico, al marco tectónico, a la litología y principalmente a la composición mineralógica, además de una similitud de las huellas geoquímicas, esto permite atribuir que el depósito de sulfuros masivos tipo Besshi, se correlaciona con los depósitos de sulfuros masivos de la división Salado, sin embargo, las características de formación y la evolución de esta unidad ha dificultado el desarrollo completo de estos depósitos, pero no se descarta la existencia de los mismos.

En la división Salado tenemos una evidente mineralización de sulfuros masivos volcanogénicos presentes en Guarumales, dentro de los esquistos micáceos negros, grises y verdes de las litologías de la subdivisión Upano, en la orilla oriental del río Paute, inmediatamente aguas abajo del puente Guarumales. La roca mineralizada está representada por esquistos micáceos blancos y verdes con más o menos pirita diseminada de granulometría fina a media (Jamielita \& Bolaños, 1993).

Los valores geoquímicos del ensayo realizado por Jamielita \& Bolaños, 1993, para muestras de este lugar dan hasta $1,32 \%$ en $\mathrm{Cu}, 7,97 \%$ de $\mathrm{Pb}$ y $12,32 \%$ de $\mathrm{Zn}$, acompañado de valores altos o anomalías de $\mathrm{Au}, \mathrm{Ag}, \mathrm{As}, \mathrm{Sb}, \mathrm{Bi}, \mathrm{Cd}, \mathrm{Ba}, \mathrm{Mo}, \mathrm{W}$ y Sn. Por lo que Hutchinson, 1980 y Franklin et al., 1981 consideran a esta mineralización como mineralización de sulfuros masivos volcanogénicos polimetálicos. 
Tabla 2. Comparación de características geológicas

\begin{tabular}{|c|c|c|}
\hline Características & vHMs tipo Kuroko & Unidad Alao-Paute \\
\hline Entorno geológico & $\begin{array}{l}\text { Secuencia volcano-sedimentaria con } \\
\text { características de arco de islas oceáni- } \\
\text { cas y cuenca marginal }\end{array}$ & $\begin{array}{l}\text { Arco insular (antearco-arco de isla } \\
\text { oceánica-retroarco) }\end{array}$ \\
\hline Marco tectónico & $\begin{array}{l}\text { Arcos de islas y dentro de ellos secto- } \\
\text { res con actividad tectónica extensional }\end{array}$ & $\begin{array}{l}\text { Arco de isla acrecionado al con- } \\
\text { tinente con intensa deformación } \\
\text { por la falla frente Baños y una } \\
\text { tectónica regional de fallas poste- } \\
\text { riores de rumbo E-NE }\end{array}$ \\
\hline Rocas encajantes & $\begin{array}{l}\text { Rocas volcánicas submarinas de com- } \\
\text { posición félsica a intermedia }\end{array}$ & $\begin{array}{l}\text { Esquistos verdes y metavolcanitas } \\
\text { con albita }+ \text { epidota }+ \text { clorita } \pm \\
\text { cuarzo }\end{array}$ \\
\hline $\begin{array}{l}\text { Características texturales y mineraló- } \\
\text { gicas de los cuerpos masivas }\end{array}$ & $\begin{array}{l}\text { Sulfuros dominantes: pirita + esfa- } \\
\text { lerita + calcopirita, yeso/anhidrita, } \\
\text { Textura bandeada con pliegues de } \\
\text { «slumping». }\end{array}$ & $\begin{array}{l}\text { Los cuerpos presentan recristali- } \\
\text { zación intensa, pirita en cristales } \\
\text { idiomorfos y subidiomorfos, } \\
\text { bandeamiento paralelo }\end{array}$ \\
\hline Características geoquímicas & $\begin{array}{l}\text { Enriquecidos en } \mathrm{Mg} \text { y } \mathrm{Zn} \text { y diminu- } \\
\text { ción del } \mathrm{Na} \text {. En los depósitos: } \mathrm{Cu}, \mathrm{Zn} \text {, } \\
\mathrm{Pb}, \mathrm{Ba}, \mathrm{As}, \mathrm{Ag}, \mathrm{Au}, \mathrm{Se}, \mathrm{Sn}, \mathrm{Bi} \text { y Fe }\end{array}$ & $\begin{array}{l}\mathrm{Fe}-\mathrm{Cu}-\mathrm{Pb}-\mathrm{Zn} \pm \mathrm{Ag} \pm \mathrm{Au}(\mathrm{sul}- \\
\text { furos masivos volcanogénicos) }\end{array}$ \\
\hline
\end{tabular}

Basado en Singer (1986a); Singer y Mosier (1986); Hoy (1995b); Litherland et al. (1994).

Tabla 3. Comparación de características geológicas

\begin{tabular}{|c|c|c|}
\hline Características & vнмs tipo Besshi & División Salado \\
\hline Entorno geológico & $\begin{array}{l}\text { Secuencia sedimentaria clástica terrí- } \\
\text { gena asociado con volcanitas marinas } \\
\text { y localmente rocas calcáreas }\end{array}$ & $\begin{array}{l}\text { Secuencia de cuenca arco de } \\
\text { isla: cuenca marginal; prisma de } \\
\text { acreción plataforma submarina } \\
\text { marginal }\end{array}$ \\
\hline Marco tectónico & $\begin{array}{l}\text { Terrenos metamórficos intensamente } \\
\text { deformados. Formación en cuencas } \\
\text { de rift, en arcos de islas o zonas trase- } \\
\text { ras de arco }\end{array}$ & $\begin{array}{l}\text { Cuenca marginal de Jurásico infe- } \\
\text { rior sobre basamento continental } \\
\text { y con granitoides de edad Jurásico } \\
\text { medio-superior }\end{array}$ \\
\hline Rocas encajantes & $\begin{array}{l}\text { Sedimentos clásticos finamente } \\
\text { laminados y tobas y/o brechas máficas } \\
\text { tholeiticas y andesíticas }\end{array}$ & $\begin{array}{l}\text { Esquistos pelíticos y metagrauva- } \\
\text { cas }\end{array}$ \\
\hline $\begin{array}{l}\text { Características texturales y mineraló- } \\
\text { gicas de los cuerpos masivas }\end{array}$ & $\begin{array}{l}\text { Sulfuros: pirita + pirrotina + calcopi- } \\
\text { rita + esfalerita } \pm \text { magnetita } \pm \text { galena; } \\
\text { Laminación muy fina y presencia de } \\
\text { pirita coloforme y framboidal }\end{array}$ & $\begin{array}{l}\text { Sulfuros diseminados predomi- } \\
\text { nantemente pirita, presenta direc- } \\
\text { ción paralelo a la esquistosidad }\end{array}$ \\
\hline Características geoquímicas & $\begin{array}{l}\text { Huella geoquímica de } \mathrm{Cu}, \mathrm{Zn}, \mathrm{Ag}, \mathrm{Ni} \\
\mathrm{Cr} \text {, Co y Co/Ni>10 halos de } \mathrm{Mn}\end{array}$ & $\begin{array}{l}\mathrm{Fe}-\mathrm{Cu}-\mathrm{Pb}-\mathrm{Zn} \pm \mathrm{Ag} \pm \mathrm{Au}(\mathrm{Sul}- \\
\text { furos masivos volcanogénicos) }\end{array}$ \\
\hline
\end{tabular}

Basado en Singer (1986a); Singer y Mosier (1986); Hoy (1995b), Litherland et al. (1994). 


\section{CONCLUSIONES}

En la unidad Alao se registra un evento de rejuvenecimiento mediante el cual se propone que los fluidos cenozoicos sean canalizados a lo largo de fallas. Se interpreta que los depósitos polimetálicos de Ag$\mathrm{Cu}-\mathrm{Zn}-\mathrm{Pb}$ relacionados al arco Alao-Paute presenta características muy favorables para el desarrollo de vHMs posiblemente de tipo Kuroko, relacionados a la formación del arco de isla, lo que se han constatado con varios depósitos muy rentables que se encuentran en explotación dentro de esta unidad. Por otro lado, tenemos la unidad Peltetec que con sus discrepancias en varios aspectos geológicos no puede presentar depósitos tipo vHMs.

La división Salado ha sido de mayor interés, ya que se ha discutido en cuanto a su evolución geológica y su entorno geológico, para el cual, basados en varios autores, interpretamos que pertenece a una cuenca marginal que se desarrolló en un ambiente extensivo, durante el funcionamiento del rift Triásico-Jurásico inferior, esta cuenca marginal tuvo el aporte de material volcánico del arco en funcionamiento Misahuallí y sedimentos terrígenos provenientes de la erosión del cratón sudamericano, por este motivo le asociamos un depósito vHMs tipo Besshi, pues este modelo es óptimo para cuencas de rift y arcos de islas o zonas traseras de arco, también tenemos una gran similitud en el marco tectónico presentando rocas metamórficas deformadas presentadas en las fajas sub horizontales de la unidad Cuyuja, otras características que favorecen a este modelo es la parte litológica con la presencia de rocas verdes de la unidad Upano, la presencia de rocas carbonatadas representadas en la unidad Cerro Hermoso y las huellas geoquímicas de $\mathrm{Fe}, \mathrm{Cu}, \mathrm{Pb}$, $\mathrm{Zn} \pm \mathrm{Ag} \pm \mathrm{Au}$, a pesar de ello, en la división Salado se encuentran sulfuros diseminados con predominancia de pirita siendo la única diferencia de mayor peso para esto modelo, ya que el modelo presenta una paragénesis de pirita + pirrotina + calcopirita + esfalerita \pm magnetita \pm galena.

Por lo tanto, esta división es óptima para presentar depósitos tipo vHMs, en especial el tipo Besshi, gracias a todas las similitudes, sin embargo, la evolución general de la división Salado no favorece a la formación de grandes depósitos o depósitos económicamente rentables para su explotación, en contraste con esto no se descarta la existencia de depósitos tipo Besshi, pero en un tamaño o proporción reducida, se necesitan estudios a detalle y una exploración más completa en la división Salado y este trabajo es la iniciativa para realizarlos.

\section{REFERENCIAS}

Aspden, J. A. y Litherland, M. 1992. Geología e historia colisional mezosoica de la cordillera Real, Ecuador. En W. G. Bonilla (ed.), Boletín Geológico Ecuatoriano, 4(1), pp. 33-53.

Baby, P., Rivadeneira, M. y Barragán, R. 2004. La cuenca Oriente: geología y petróleo. Convenio IFEA, IRD, Petroproducción, pp. 295.

Best, M. 2003. Igneous and metamorphic petrology (2th ed). Melbourne: Blackwell.

Bucher, K. and Grapes, R. 2011. Petrogenesis of metamorphic rocks (8th ed). London: Springer.

Cochrane, R. S. 2013. U/Pb thermochronology, geochronology and geochemistry of NW South America: rift to drift transition, active margin dynamics and implications for the volume balance of continents (Tesis doctoral). Université de Genève, Faculté des sciences de l'Université de Genève, Cap (Sudáfrica).

Cox, D. P. 1986. Descriptive model of Besshi massive sulfide. In Cox, D. P., and Singer, D. A. (eds.), Mineral deposit models: U.s. Geological Survey Bulletin, 1693, p. 136.

Cox, D. P. and Singer, D. A. 1986. Mineral deposit models: U. S. Geological Survey Bulletin, 1693, p. 379.

Filipek, L. H., Nordstrom, D. K. and Ficklin, W. H. 1987. Interaction of acid mine drainage with waters and sediments of West Squaw Creek in the West Shasta mining district, California. Environmental Science and Technology, 21(4), 388-396.

Litherland, M. 1988. Second annual report (March 1986-March 1987) of the Cordillera Real Geological Research Project, Ecuador. British Geological Survey. Keyworth (in english) and of INEMIN, Quito (in spanish).

Litherland, M., Aspden, J. and Jemielita, R. 1994. The metamorphic belts of Ecuador (first ed.). Keyworth: NERC.

Pratt, W., Duque, P. and Ponce, M. 2005. An autochthonous geological model for the eastern Andes. Tectonophysics, 399, 251-278.

Prodeminca. 2000. Evaluación de distritos mineros del Ecuador, sulfuros masivos alojados en vulcanitas, vol. 3. Quito: UCP Prodeminca Proyecto Mem Birf 36-55 Ec.

Singer, D. A. 1986a. Descriptive model of Cyprus massive sulfide. In Cox, D. P., and Singer, D. A. (eds.), Mineral deposit models: U. S. Geological Survey Bulletin, 1693, p. 131.

Singer, D. A. 1986b. Descriptive model of Kuroko massive sulfide. In Cox, D. P., and Singer, D. A. (eds.), Mineral deposit models: U. S. Geological Survey Bulletin, 1693, pp. 189-190.

Spikings, R., Cochrane, R., Villagómez, D., Van der Lelij, R., Vallejo, C., Winkler, W., and Beate, B. 2015. The 
geological history of northwestern South America: from Pangaea to the early collision of the Caribbean
Large Igneous Province (290-75 Ma). Gondwana Research (GR-01278), p. 45. 